\title{
Race et médecine
}

\section{Une vieille histoire}

Claude-Olivier Doron

$>$ Cet article propose de prendre un peu de distance vis-à-vis du retour actuel de la notion de « race » dans le champ biomédical, en examinant le lien intime qui existe entre pensée médicale et problématique de la race lors de l'émergence du concept naturaliste de « race » aux XvIII ${ }^{e}-$ $\mathrm{XIX}^{\mathrm{e}}$ siècles. II montre comment l'analogie avec les pathologies a été mobilisée alors par les naturalistes pour penser la « race » comme altération d'une identité d'origine, à travers, d'une part, le modèle des tempéraments maladifs, d'autre part celui des maladies héréditaires. <

La problématique de la « race » a fait, depuis quelques années, un retour controversé dans le champ biomédical. Qu'il s'agisse de tentatives visant à interroger les liens entre des polymorphismes génétiques plus fréquents selon l'appartenance à certains « groupes d'ascendance » et des risques plus marqués de développer certaines maladies [1,2] ; qu'il s'agisse d'études visant à identifier certains polymorphismes responsables de réponses différentes aux médicaments, réputés caractéristiques de certaines « races », avec pour objectif final de développer des traitements adaptés à ces populations [3, 4]. Dans tous les cas, le concept de « race », entendu soit comme ensemble populationnel partageant dans des proportions significatives des marqueurs génétiques informatifs sur une origine commune, soit comme ensemble construit à partir des déclarations des personnes, est réputé pertinent pour la recherche et la clinique médicales ${ }^{1}$. À dire vrai, cette insistance de la « race » dans les travaux biomédicaux ne sera considérée comme un retour surprenant que par ceux qui ont cru aux déclarations un peu hâtives de certains scientifiques qui annonçaient après-guerre la disparition du concept de « race », réputé non scientifique et infondé. Sous ce discours, qui ne concernait en vérité qu'une conception

${ }^{1}$ Cela ne va pas sans susciter de très nombreux débats (voir par exemple [2], pp. 147-64).

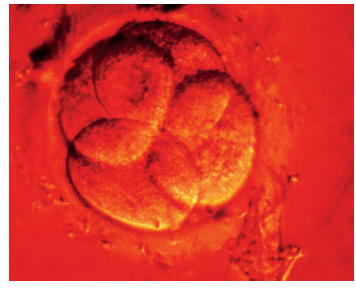

Laboratoire SPHERE

(UMR7219)/ Centre Canguilhem, Université Paris VII, 207, rue La Fayette, 75010 Paris, France. colivierdoron@gmail.com

bien précise de la « race $»^{2}$, le concept perdurait et tendait même à se renouveler à travers les analyses de la génétique des populations humaines, I'hémotypologie $[6,7]$ et les projets visant à cartographier les polymorphismes génétiques humains $[2,5,8]$. À ce renouvellement, la médecine a d'ailleurs largement contribué.

Le fait est donc là : dans le constat de relations régulières entre problématique de la race et pensée biomédicale. Nous ne chercherons pas ici à retracer toute l'histoire, extrêmement complexe, de ces relations; nous proposons simplement de montrer, très schématiquement, combien elles sont anciennes. Elles commencent au moment même de l'émergence du concept naturaliste de « race » et tiennent alors au fait que, dès leurs premières élaborations, l'analyse médicale et l'analogie avec certaines pathologies ont été mobilisées pour penser la « race». On pourrait aussi bien montrer cette affinité par une analyse institutionnelle, puisque ce sont souvent des médecins qui ont contribué à la « science des races ». Mais nous examinerons plutôt certains aspects de cette affinité en termes d'histoire des concepts. II ne s'agira donc en aucun cas de chercher à tracer une continuité linéaire entre la manière dont des conceptualisations de la « race », d'ailleurs très diverses, se trouvent aujourd'hui impliquées dans la recherche biomédicale et la situation historique que nous allons analyser. Même si nous pensons que certains éléments de cette situation peuvent éclairer la situation actuelle, il faudrait de nombreuses pages pour établir là où sont les continuités et là où se situent les indéniables ruptures ${ }^{3}$. Ici, nous chercherons simple-

\footnotetext{
${ }^{2}$ Après la Deuxième Guerre mondiale, notamment dans le cadre des déclarations de l'UNESCO, de nombreux travaux, en particulier issus d'anthropologues culturels, ont critiqué le concept de « race » comme relevant de l'idéologie et n'ayant guère de valeur scientifique. Divers généticiens ont, quant à eux, rattaché la « race » à une analyse typologique, statique et discrète des différences humaines à laquelle il convenait de substituer une analyse populationnelle, dynamique et clinale. Mais, comme l'ont montré divers travaux, cette «substitution » ne s'est pas faite au prix d'un abandon du concept de « race», tout au contraire (voir par exemple [5], pp. 17-73). Le fait est que la conception typologique et discrète des races humaines n'a jamais été qu'une manière d'utiliser le concept de « race », critiquée dès le xixe siècle. Elle n'a jamais fait le tout des conceptualisations de la « race », ce qui explique que d'autres conceptualisations puissent perdurer alors même qu'elle a été récusée.

${ }^{3}$ Nous nous réservons la possibilité d'y revenir dans d'autres articles.
} 
ment à démontrer deux thèses : (1) les concepts médicaux ont joué un rôle essentiel dans la constitution d'une réflexion sur les races humaines aux XVIII et $\mathrm{XIX}^{\mathrm{e}}$ siècles; (2) ce rôle a été particulièrement important lorsqu'il s'est agi de penser la formation de variétés héréditaires différenciées à partir d'une origine unique.

\section{Le problème de l'altération : la formation des races humaines au XVIII ${ }^{\mathrm{e}}$ siècle}

L'historiographie des savoirs sur les races humaines a largement envisagé la question des races sous l'angle de l'altérité. L'histoire du concept de « race » serait à chercher du côté de travaux visant à caractériser un ensemble de différences radicales, originelles et inaltérables dans I'humanité et à établir des classifications fixes sur la base de ces différences. Tendanciellement, le concept de « race »logerait du côté de l'affirmation qu'il existe plusieurs espèces au sein du genre humain, renvoyant à des origines différentes (polygénisme) et définissant des groupes radicalement séparés ${ }^{4}$. Dans une telle lecture, le rôle de la médecine est relativement secondaire, même s'il n'est pas négligeable. Ce que la médecine apporte, c'est un savoir des différences radicales inscrites dans les corps et, en particulier, dans les tempéraments des différentes races [9]. Une histoire plus attentive aux usages réels du concept de « race » en histoire naturelle dans la seconde moitié du XVIII et la première moitié $d u x^{e} x^{e}$ siècle vient nuancer ces analyses. D'une part, le concept de « race » a été très largement élaboré par la tradition monogéniste ${ }^{5}$ (de Buffon à Prichard, en passant par Blumenbach, Kant et Lawrence) pour caractériser des différences relativement constantes, transmises par hérédité et inscrites dans la généalogie, sans remettre en cause l'unité de l'espèce et la communauté d'origine. La race définit pour la plupart des auteurs un niveau taxinomique en-deçà de l'espèce : c'est une variété qui se transmet par la génération. D’autre part, sa dimension la plus caractéristique ne tient pas tant dans sa dimension taxinomique per se (la « race » n'est pas au départ une catégorie taxinomique, contrairement au genre, à l'espèce ou à la variété) que dans le fait qu'elle suppose une classification fondée sur la généalogie et les rapports de parenté. La race est en effet intiment liée à l'introduction d'un mode de raisonnement généalogique en histoire naturelle [10]. Elle désigne un ensemble de caractères qui se transmettent de génération en génération et fondent des lignées relativement différenciées à partir d'une identité d'origine, dans le cadre de l'espèce.

Le grand problème auquel s'affrontent la plupart des premiers théoriciens de la race, ce n'est pas tant celui de l'altérité que celui de l'altération. II s'agit de comprendre comment, à partir d'une identité d'origine, ont pu se former des lignées nettement différenciées ; comment des différences se transmettent et s'accumulent jusqu'à produire des

\footnotetext{
${ }^{4}$ Nous résumons ici à grands traits les principes généraux de nombreuses histoires de la « race ». II est facile de voir en quoi ils imprègnent la vision de la « race » dénoncée au sortir de la Deuxième Guerre mondiale : les races seraient conçues comme des entités discrètes, statiques, servant de base à une taxinomie fixe des différences humaines.

${ }^{5}$ Le monogénisme, en ce sens, n'a évidemment rien à voir avec les maladies héréditaires dites monogéniques. II désigne la position qui affirme que l'ensemble des races humaines sont issues d'une souche unique, contre une position «polygéniste » qui affirme qu'il s'agit d'espèces distinctes, issues d'origines distinctes.
}

distinctions en apparence radicales. Or, pour penser ce processus d'altération, les naturalistes vont mobiliser deux modèles distincts. D'une part, celui de l'élevage, dans la mesure où l'élevage permet d'étudier comment se forment des lignées distinctes par accumulation de différences sélectionnées d'une génération à l'autre à partir d'une origine unique. D'autre part, et c'est sur ce point que nous insisterons, celui de la pathologie et des altérations plus ou moins morbides étudiées par la médecine. Ici, donc, la médecine n'occupe plus un rôle accessoire dans la conceptualisation de la race mais une place décisive. Dès lors qu'il s'agit de penser les races comme des altérations d'une norme d'origine, le jeu entre le normal et le pathologique, typique du raisonnement médical, devient essentiel : il fournit des concepts et des analogies. Du même coup s'instaure une tension dans laquelle le $x_{\mathrm{X}} \mathrm{x}^{\mathrm{e}}$ siècle se débattra régulièrement : comment établir une distinction entre variétés normales et variétés morbides au sein de l'espèce humaine [11]?

\section{Les idiosyncrasies de l'espèce : races et tempéraments maladifs}

Comme l'écrit le médecin montpelliérain Antoine Taillefer, « autant un individu isolé est sujet à des idiosyncrasies, autant l'espèce en général est forcée de se plier à des variétés accidentelles qui l'éloignent, par des aberrations plus ou moins remarquables, du type universel de l'espèce $\gg^{6}$. C'est sans doute le modèle le plus ancien, qui connaît un regain de vigueur au milieu du xvIII siècle avec le regain des thèses hippocratiques: de même que chaque individu possède un tempérament caractéristique, plus ou moins équilibré en fonction de l'influence du climat et des choses non naturelles, de même, chaque variété dans l'espèce humaine présente un tempérament particulier. Si ce modèle est ancien, il est par contre très original de considérer qu'il y a une idiosyncrasie propre au type de l'espèce, dont les variétés s'écartent plus ou moins. Dans tous les cas, les altérations des humeurs conduisent à des constitutions nettement différenciées entre individus comme entre races.

Ce modèle permet de définir certaines constitutions propres à certaines variétés comme quasi-morbides mais devenues naturelles. Lorsque le médecin et botaniste Pierre Barrère (vers 1690-1755) cherche à expliquer la cause physique de la couleur des Nègres, il en rend compte par la surabondance dans leur sang d'une substance noire comparable à la bile. Ce déséquilibre se traduit par d'autres caractéristiques typiques d'une

\footnotetext{
${ }^{6}[12]$, p. 5.
} 
bile surabondante: "la force et la célérité du pouls, l'extrême lubricité et les autres passions fougueuses et surtout la chaleur considérable de la peau $»^{7}$. Un compte rendu de son ouvrage accroît encore l'analogie avec une constitution morbide: "tous les jours, dans la jaunisse, on observe parmi nous que la bile s'épanche jusqu'à donner son jaune à la peau. Et dans l'ictère noir, les épanchements de la bile sur la peau sont noirs [...] Ne pourrait-on pas regarder la couleur des Nègres comme un ictère naturel ? $\gg^{8}$. Buffon lui-même admet que la couleur des Nègres trouve pour partie son explication dans « une espèce de jaunisse » dont souffrent l'ensemble des enfants humains à la naissance mais qui «chez les Blancs n'a qu'un effet passager et ne laisse à la peau aucune impression; dans les Nègres, au contraire, elle donne à la peau une couleur ineffaçable »". Mais c'est le philosophe Cornelius de Pauw (1739-1799), auteur des très controversées Recherches philosophiques sur les Américains (1768), qui résume le mieux cette analogie entre état morbide et constitution des Nègres. Sa description ressemble à un tableau clinique : « leur pouls est presque toujours vif et accéléré et leur peau [...] paraît échauffée : aussi leurs passions sont-elles fougueuses, immodérées, excessives et n'obéissent à aucun frein de la raison [...] comme ils ne peuvent se gouverner eux-mêmes, ceux qui les gouvernent en font d'excellents esclaves. Les organes les plus délicats [...] de leur cerveau ont été détruits ou oblitérés par le feu de leur climat natal ${ }^{10}$. De Pauw est celui qui pousse le plus loin l'analogie entre production des différentes variétés dans l'espèce humaine et tempéraments maladifs issus de climats délétères. Cette thèse est exprimée en particulier à propos des Indiens, qui constituent à ses yeux une dégénération radicale de l'espèce en raison du climat particulièrement dégradé des Amériques. Les Indiens présentent une constitution faible, humide et froide qui les rapproche des femmes; leur sang est plus visqueux, au point que la syphilis leur est «naturelle », c'est « une affection de leur tempérament [plus] qu'une qualité morbifique $»^{11}$. À cette constitution défectueuse correspond un abrutissement intellectuel et moral.

\section{La transmission des altérations}

Mais ces altérations du tempérament ne suffisent pas à faire une race. Celle-ci suppose un mécanisme de transmission des altérations à travers les générations. C'est ce que souligne Buffon à propos des Nègres : la jaunisse et l'influence actuelle du climat «ne [...] paraissent être que des causes occasionnelles de la noirceur et non pas la cause première [...] II est certain que le germe de la noirceur est communiqué des parents aux enfants ${ }^{12}$. C'est donc un principe de transmission héréditaire des altérations qui rend compte de la formation des races. Buffon y insiste dans la conclusion de son article sur les Variétés dans l'espèce humaine : ces variétés ne deviennent «variétés de l'espèce [que] parce qu'elles sont devenues plus générales, plus sensibles et plus constantes par l'action

${ }^{7}[13]$.

${ }^{8}[14]$, p. 1650.

9 [15], pp. 522-3.

${ }^{10}[16]$, T. I, p. 182

${ }^{11}[16]$, T.I, p. 46.

$12[15]$, p. 253. Nous soulignons. continuée des mêmes causes; qu'elles se sont perpétuées et qu'elles se perpétuent de génération en génération comme les difformités ou les maladies des pères et des mères passent à leurs enfants $\gg^{13}$. Le même constat est établi par De Pauw qui s'oppose ainsi aux thèses polygénistes sur l'origine distincte des Blancs et des Noirs. La dégénération qui affecte les Noirs se transmet dans leur semence et passe d'une génération à l'autre, si bien que la couleur, variété accidentelle liée au climat, se fixe peu à peu dans leur lignée. "Si l'on voulait révoquer en doute que la substance qui sert à la reproduction puisse ou se charger, ou entraîner avec elle un levain venimeux, on n'aurait qu'à citer cette longue et affligeante liste de maladies héréditaires qui se perpétuent plus opiniâtrement dans les familles qu'il ne serait à souhaiter pour le bien de l'humanité $\gg^{14}$. On le voit, dès lors qu'il s'agit d'envisager comment des variétés accidentelles font race et se transmettent, un modèle surgit aussitôt : celui des maladies héréditaires ${ }^{15}$. Là encore, l'analogie avec la pathologie joue à plein.

Il faut distinguer deux modèles qui permettent de rendre compte de ce processus de déviation à travers les générations comme mécanisme de formation des races.

\section{Premier modèle : les races, dégénérations du type spécifique}

Selon Frédéric Cuvier (1773-1838), la dégénération caractérise toute altération héréditaire produisant des «modifications organiques [qui] ne sont plus individuelles [mais] deviennent inhérentes à la nature intime des êtres et se perpétuent de génération en génération [...] C'est ainsi que se forment les variétés et les races ${ }^{16}$. Ce modèle de la dégénération a été introduit par Buffon, qui s'inspirait de la manière dont les éleveurs interprétaient la perte progressive des qualités originaires chez les races transplantées. Aux yeux de Buffon, l'espèce présente une forme spécifique qui se transmet à travers les générations. Ce type subit des altérations du fait de l'influence des conditions d'existence qui se transmettent d'une génération à l'autre, accroissant le processus de déviation et l'inscrivant au plus profond des corps. C'est ce processus qui explique la formation des races [20]. Cette notion de « dégénération », notons-le, contient une évaluation qualitative négative du processus de déviation, qui tend

\footnotetext{
${ }^{13}$ [15], p. 530. Nous soulignons.

14 [16], T.II, p. 26. Nous soulignons.

${ }^{15}$ Sur l'histoire du problème des maladies héréditaires et leur rôle dans la formation du concept biologique d'hérédité, voir [17]. Rappelons que nous nous situons précisément dans la période - 1750-1850 - qui voit le concept biologique se forger progressivement [18]. Les auteurs que nous avons cités - Buffon, Maupertuis, Blumenbach, Prichard... - sont autant d'auteurs essentiels dans l'élaboration du concept biologique d'hérédité.

${ }^{16}$ [19].
} 
à l'inscrire en continuité avec le pathologique ${ }^{17}$. Cette dimension est encore accrue par l'interprétation qu'en donne le naturaliste allemand Johann Friedrich Blumenbach (1752-1840). Pour Blumenbach, les êtres vivants sont caractérisés par une force formatrice qui impose à la matière organique une forme qui est celle de l'espèce et des ascendants [21]. Ce nisus formativus s'exprime en particulier dans le processus de génération et le développement de l'embryon. Or cette force qui « conserve la forme et l'habitude [...] de toutes les espèces distinctes [...] peut dévier de diverses manières de sa direction propre et déterminée ». Ces déviations produisent les monstruosités, c'est-à-dire toute « difformité frappante contre-nature »; les hermaphrodites; les hybrides; et «les races et variétés ${ }^{18}$. Les races sont donc pensées comme des écarts, plus ou moins marqués, du type originel, au même titre que les monstruosités. Elles sont des anomalies de l'espèce dans lesquelles celle-ci « se dégrade de sa conformation primitive ». Un tel schéma rend particulièrement aigu le problème de la détermination du type originel. Pour Blumenbach, c'est la race caucasienne qui constitue «l'espèce primitive. Celle-ci diverge en deux directions [...] d'un côté dans l'éthiopienne, et de l'autre dans la Mongole $»^{19}$. La race caucasienne est donc la plus proche du type normal ; les races éthiopienne et mongole définissent le maximum de dégradation de l'espèce. Il est intéressant de noter que Blumenbach estime par ailleurs que des races peuvent être produites par « $[d]$ es particularités héréditaires [...] issues d'un tempérament maladif ». L'organisation s'habitue alors à ce point à la maladie, au fil des générations, qu'elle devient « comme une seconde nature » et forme des races constantes, comme dans le cas des lapins albinos ${ }^{20}$. Ici, la frontière entre pathologie et histoire naturelle se brouille singulièrement.

\section{Second modèle : l'erreur héréditaire}

Si le premier modèle insiste sur le rôle joué par les agents extérieurs dans la production des déviations et sur la forme spécifique comme objet de la déviation, le second modèle, issu des travaux du naturaliste anglais Henry Baker (1698-1774) et de Maupertuis (1698-1759), s'en distingue quelque peu. D'abord, à l'influence des agents extérieurs se substitue une tendance interne à la variation qui est le fruit du hasard. Pour Maupertuis, le processus de génération naturelle est marqué par des « accidents », des « erreurs », qui produisent des « individus fortuits ». Ces individus fortuits sont transformés, soit par l'élevage, soit par le hasard, à travers la répétition des générations, en « races constantes ». Aux yeux de Maupertuis, les maladies héréditaires et les malformations familiales offrent une analogie parfaite avec ce phénomène. Lorsqu'il prend le cas du Nègre blanc (un Noir albinos) pour expliquer le processus général de formation des races, il note, à la suite d'un auteur anglais : «cette blancheur [est] comme une maladie de la peau; c'est [...] un accident mais un accident qui se perpétue et qui subsiste pendant plusieurs générations [...]. Qu'on prenne cette blancheur

\footnotetext{
${ }^{17}$ II faut noter cependant que, chez Buffon comme chez De Pauw, elle va de pair avec la définition de critères permettant de distinguer fermement entre « races » et variétés morbides ou monstrueuses (crétins, albinos, hermaphrodites, etc.). Ce critère tient précisément dans la possibilité ou non de se reproduire sur plusieurs générations.

18 [22], pp. 24-25.

19 [23], p. 269.

$20[23]$, p. $202-3$.
}

pour une maladie ou pour tel accident qu'on voudra, ce ne sera jamais qu'une variété héréditaire qui se confirme ou qui s'efface par une suite de générations $\gg^{21}$. Cette transmission de l'erreur hasardeuse comme modèle de production des races se retrouve dans les réflexions que Henry Baker consacre au cas Edward Lambert, « l'homme porcépic », sans cesse cité ensuite pour montrer comment les races, mêmes les plus différentes en apparence, peuvent être issues d'une origine unique. Lambert était atteint d'une forme grave d'ichthyosis qui lui donnait un aspect monstrueux ; il transmit sa maladie à ses six enfants. La thèse de Baker est qu'il «semble hors de doute qu'une race d'homme puisse se propager à partir de cet homme [...] Si cela arrive [...] et qu'alors l'origine accidentelle est oubliée, il n'est pas improbable qu'elle soit considérée comme une espèce différente dans le genre humain. Une considération qui pourrait presque nous laisser penser que, si le genre humain a été produit à partir d'une souche unique et identique, la peau noire des Nègres et d'autres différences du même type ont pu apparaître originellement pour les mêmes causes accidentelles $»^{22}$. On voit que le modèle des maladies héréditaires permet ici encore de fonder une explication monogéniste de la formation des races. On retrouvera cet exemple chez les médecins et naturalistes anglais William Lawrence (1783-1867) [27] et James Cowles Prichard (1786-1846) [28], qui, I'un comme l'autre, insistent sur la tendance interne à la variabilité et à la transmission de ces accidents, illustrées par les maladies héréditaires, pour rendre compte de la formation des races sans recourir à l'influence du climat. D'autre part, en particulier chez Maupertuis, ce modèle de l'erreur se préoccupe peu de la forme du type et de sa déviation : il repose sur une conception beaucoup plus moléculaire et localisée de l'accident, qui affecte tel ou tel caractère en particulier et non la forme spécifique en général. C'est ce qui rend ce modèle beaucoup plus « moderne » que celui de la dégénération du type et explique sa reprise favorable par la génétique post-mendélienne [29].

Pour être complet, il aurait fallu décrire un troisième modèle, où la race est moins perçue comme une déviation d'une identité d'origine que comme un arrêt de développement dans la réalisation d'un type. Là encore, en effet, le langage médical va jouer à plein ${ }^{23}$. Mais nous espérons avoir suffisamment démontré deux choses : (1) l'intime relation entre le concept de « race » et la

\footnotetext{
${ }^{21}$ [24], pp. 150-1. Voir aussi [25].

22 [26], p. 23.

${ }^{23}$ Ce modèle de l'arrêt de développement s'élabore dès Cornelius de Pauw. On le trouve parfaitement exprimé par le naturaliste Armand De Quatrefages (1810-1892) pour qui « le nègre est un blanc dont le corps acquiert la forme définie de l'espèce mais dont l'intelligence entière s'arrête en chemin » ([30] p. 757), « le nègre adulte [est] un être dont l'intelligence est restée, par une sorte d'arrêt de développement, au point où nous l'observons chez les adolescents de la race blanche » ([31], p. 429).
} 
pensée médicale aux XVIII et $x I x^{e}$ siècles; (2) le fait que cette relation a été particulièrement essentielle quand il s'est agi de rendre compte de la production de différences relativement constantes dans l'identité et de penser les races comme des altérations d'une identité de référence, selon la tradition monogéniste. Dans ce cas, qu'il se soit agi de penser cette altération à travers des tempéraments différenciés ou sa transmission à travers l'analogie des maladies héréditaires, les concepts médicaux ont offert des modèles décisifs. Quel a été le devenir de ces modèles par la suite? Les transformations considérables introduites par le darwinisme, qui valorise les variations individuelles héréditaires et met à mal la notion de «type spécifique », ont-elles suffi à les ébranler entièrement? Que devient le jeu du normal et du pathologique quand la norme à laquelle rapporter la variation n'est plus un type de référence mais une fréquence statistique dans une population et/ou une valeur adaptative? S'il nous était impossible d'aborder ces questions $\mathrm{ici}^{24}$, nous espérons que cet article permettra de mieux repérer où, au départ, se sont situés les points de contact entre race et médecine. Car c'est en établissant plus clairement l'histoire d'un problème, a fortiori aussi surchargé d'enjeux que celui-ci, qu'on peut espérer mieux repérer ensuite où se situent vraiment les discontinuités et les continuités dans la manière dont il se pose aujourd'hui, et comment il convient de le traiter. $\diamond$

\section{SUMMARY}

Race and medicine

In this article, I argue that the problematic of "race and medicine", which has been the object of many recent debates, has a long history that it may be useful to understand better. I show more specifically that, from the very first uses of the concept of "race" in natural history during the XVIII th century, medical concepts and analogies served as important models. These medical models were especially useful to analyze "races" as alterations from an original identity. Different analogies are studied here. 1. The analogy between races' peculiar temperaments and morbid alterations of human constitution. 2. The analogy between the transmission of the alterations along generations and hereditary diseases. In this second analogy, I differentiate between two models: the degeneration of the human type and the transmission of a molecular alteration of one character. $\diamond$

\section{LIENS D'INTÉRÊT}

L'auteur déclare n'avoir aucun lien d'intérêt concernant les données publiées dans cet article.

\section{RéFÉRENCES}

1. Holden C. Race and medicine. Science $2003 ; 302: 594-6$.

2. Jordan B. L'humanité au pluriel. La génétique et la question des races. Paris : Seuil, $2008: 228$ p.

3. Evans WE, Relling MV. Moving towards individualized medicine with pharmacogenomics. Nature $2004 ; 429: 464-8$.

4. Daar AS, Singer PA. Pharmacogenetics and geographical ancestry. Nat Genet $2005 ; 6: 241-6$.

5. Reardon J. Race to the finish: identity and governance in the age of genomics. Princeton :

Princeton University Press, $2004: 256$ p.

6. Boyd WC. Genetics and the races of man. Boston : Little Brown and Cie, $1950: 454 \mathrm{p}$.

\footnotetext{
${ }^{24}$ Pour un début de discussion sur certains de ces points, voir [32].
}

7. Ruffié J. Place actuelle de l'hémotypologie dans la recherche anthropologique. Bulletins et Mémoires de la Société d'Anthropologie de Paris $1965 ; 7: 287-99$.

8. Jordan B. ADN, ascendance génétique et « race » sociale : l'apport des Snip. Med Sci (Paris) $2010 ; 26: 215-8$.

9. Williams $\varepsilon A$. The physical and the moral. Cambridge : Cambridge University Press, $1994: 282 \mathrm{p}$.

10. Doron CO. Race and genealogy. Buffon and the formation of the concept of race. Humana Mente 2012 ; $22: 75-109$.

11. Morel BA. Traité des dégénérescences physiques, intellectuelles et morales de l'espèce humaine, Paris : Baillière, $1857: 692$ p.

12. Taillefer A. L'influence du climat sur l'espèce humaine, essai physiologique. Montpellier : Martel Ainé, 1783.

13. Barrère P. Dissertation sur la cause physique de la couleur des Nègres, de la qualité de leurs cheveux, et de la dégénération de l'un et de l'autre. Paris : Simon, 1741.

14. Compte rendu de la Dissertation sur la cause physique de la couleur des Nègres. Mémoires pour l'histoire des sciences et des beaux-arts. Paris : Chaubert, 1742 : 1647-57.

15. Buffon, Georges-Louis Leclerc. Variétés dans l'espèce humaine. Histoire naturelle, générale et particulière, avec la description du cabinet du Roy. Paris : Imprimerie Royale, T. III, 1749 : 371-530.

16. De Pauw C. Recherches philosophiques sur les Américains. T. I et II. Berlin : G.J. Decker, $1768-1769: 326$ p. et 366 p.

17. Lopez-Beltrán C. Human heredity (1750-1860), Ph.D. Thesis. London : King's College, 1992.

18. Rheinberger HJ, Müller-Wille S (eds). Heredity produced. At the crossroads of biology, politics and culture. Cambridge : MIT Press, 2007: $496 \mathrm{p}$.

19. Cuvier F. Dégénération. Encyclopédie des gens du monde, T. VII (1). Paris : Treuttel et Würtz, $1836: 672-3$.

20. Buffon, Georges-Louis Leclerc. Le cheval. Histoire naturelle, générale et particulière, avec la description du cabinet du Roy. Paris : Imprimerie Royale, T. IV, 1753 : 174-258.

21. Blumenbach JF. Über den Bildungstrieb. Göttigen: J.C. Dieterich, 1789 : 108 p.

22. Blumenbach JF. Manuel d'histoire naturelle, T. I, trad. fr. S. Artaud. Paris: Levrault, an XI-1803: $526 \mathrm{p}$.

23. Blumenbach JF. De generis humani varietate native (Göttingen, 1795) $3^{e}$ ed. Trad. angl. T. Bendyshe. In : Anthropological treatises. London: Longman Green, $1865: 406$ p.

24. Moreau de Maupertuis PL. Vénus physique. Paris : [sn], 1745 : 194 p.

25. Moreau de Maupertuis PL. Lettre XIV sur la génération des animaux Fuvres de Maupertuis, T. II, $2^{\mathrm{e}}$ ed. Lyon : Bruyset, 1768 : 299-314.

26. Baker H. A supplement to the account of a distempered skin. Philosophical Transactions of the Royal Society, vol. XLIX (1), London: Davis and Reimer, $1756: 21-4$.

27. Lawrence W. Lectures on physiology, zoology and the natural history of man. Salem : Foote and Brown, $1828: 494 \mathrm{p}$.

28. Prichard JC. Researches into the physical history of mankind. London: Arch, $1813: 558 \mathrm{p}$.

29. Jacob F. La logique du vivant. Paris: Tel Gallimard, 1995 (1970) : 354 p.

30. De Quatrefages A. La Floride. Revue des Deux Mondes 1843 ; $13^{e}$ année (1): 733-73.

31. Discussion sur la perfectibilité des races. Bulletins de la Société d'anthropologie de Paris, T. I, Paris : Masson, 1860 : 419-37.

32. Gannett $L$. The normal genome in twentieth-century evolutionary thought. Studies in History and Philosophy of Biological and Biomedical Sciences $2003 ; 34: 143-85$.
TIRÉS À PART

C.0. Doron 